PROCEEDINGS OF THE

AMERICAN MATHEMATICAL SOCIETY

Volume 138, Number 10, October 2010, Pages 3495-3499

S 0002-9939(10)10379-7

Article electronically published on May 10, 2010

\title{
ON A RESULT OF FALTINGS VIA TIGHT CLOSURE
}

\author{
TIRDAD SHARIF
}

(Communicated by Bernd Ulrich)

\begin{abstract}
Using a result in the theory of tight closure on F-rational rings, we prove a criterion for local rings of positive prime characteristic to be complete intersections. As an application of our criterion, we give a new and simple proof for an extension of an algebraic result of Faltings that was used by Taylor and Wiles for a simplification of the proof of the minimal deformation problem.
\end{abstract}

\section{INTRODUCTION}

The theory of tight closure was created by Melvin Hochster and Craig Huneke. An important notion in the theory of tight closure is the notion of F-rationality. The main purpose of this paper is to use a result of Hochster and Huneke in 3 , on $F$-rational rings to prove a criterion for local rings of positive prime characteristic to be complete intersections; see Theorem 2.5 for the precise statement.

In the appendix of [7, Gerd Faltings proved an algebraic result that was used by Richard Taylor and Andrew Wiles to reprove the minimal deformation problem by a simpler method. As an application of Theorem 2.5, we give a simple proof for an extension of Faltings result; see Proposition 2.8, Our method to prove this result is completely new and fundamentally different from that of Faltings, in which he simplifies Taylor and Wiles's argument.

In the following we recall the minimal deformation problem very briefly.

The modularity conjecture for semistable elliptic curves depends on a critical conjecture of Wiles that was proved in [7, 8]; see Conjecture (2.16) of [8].

Let $\mathcal{D}$ be a deformation theory and let $R_{\mathcal{D}}$ and $T_{\mathcal{D}}$ be the universal deformation and Hecke algebras associated to $\mathcal{D}$, respectively. The universal property of $R_{\mathcal{D}}$ implies that there is a ring homomorphism $\varphi_{\mathcal{D}}: R_{\mathcal{D}} \rightarrow T_{\mathcal{D}}$. Wiles's conjecture asserts that $\varphi_{\mathcal{D}}$ is an isomorphism. When $\mathcal{D}$ is a minimal deformation theory, then the above conjecture is called the minimal deformation problem. In this case, the Hecke algebra associated to $\mathcal{D}$ is of minimal level. Wiles's method in 8 , to prove the above conjecture is closely related to showing that the minimal Hecke algebras are complete intersections, proved by Taylor and Wiles in [7].

Received by the editors April 28, 2009 and, in revised form, January 13, 2010.

2010 Mathematics Subject Classification. Primary 13A35, 14M10.

Key words and phrases. Complete intersection algebras, deformation algebras, Hecke algebras, tight closure.

The author was supported by a grant from IPM (No. 83130311).

(C)2010 American Mathematical Society 
For different types of deformation theories and their relations, see Chapters 1 and 2 of [8]. We refer the interested reader to [6] for an elegant exposition of the above matters.

\section{Definitions, NOTATiOn AND ThE MAin THEOREM}

Throughout this paper all rings are commutative and Noetherian and all modules are finite (that is, finitely generated). We use the notation $\nu_{R}(M)$ and $\ell_{R}(M)$ for the minimal number of generators of $M$ and the length of $M$ over $R$, respectively.

Lemma 2.1. Let $\eta:(A, \mathfrak{n}) \rightarrow(Q, \mathfrak{m})$ be a local ring homomorphism such that $\operatorname{dim} A=\operatorname{dim} Q=t, Q$ is regular and the induced homomorphism $\bar{\eta}: A / \mathfrak{n} \rightarrow Q / \mathfrak{m}$ is an isomorphism. Let $x_{1}, \ldots, x_{t}$ be a system of parameters for $A$ and let $J$ be an ideal of $Q$. Assume that for some integers $n$ and $d$ such that $n>d^{t} t^{t-1}$, we have

(a) $\ell_{Q}\left(Q /\left(J+\left(x_{1}^{n}, x_{2}^{n}, \ldots, x_{t}^{n}\right) Q\right)\right) \geqslant \ell_{A}\left(A /\left(x_{1}^{n}, x_{2}^{n}, \ldots, x_{t}^{n}\right)\right)$

(b) $\mathfrak{m}^{t d n} \subseteq J+\left(x_{1}^{n}, x_{2}^{n}, \ldots, x_{t}^{n}\right) Q$.

Then $J \subseteq \mathfrak{m}^{d+1}$. If, in addition, $\ell_{Q}(Q /(J+I Q))=d$ for an ideal $I$ of $A$, then $J \subseteq I Q$.

Proof. Let $\mathfrak{q}=\left(x_{1}, x_{2}, \ldots, x_{t}\right)$ and for simplification write $\mathfrak{q}^{[n]}=\left(x_{1}^{n}, x_{2}^{n}, \ldots, x_{t}^{n}\right)$ when $n \geqslant 2$. Let $B=Q / J$ and choose $u$ in $J$ not in $\mathfrak{m}^{d+1}$. Consider the following exact sequence in which $c=t d n$ and $\alpha$ is the homothety by element $u$ :

$$
0 \longrightarrow \operatorname{Ker}(\alpha) \longrightarrow Q / \mathfrak{m}^{c} \stackrel{\alpha}{\longrightarrow} Q / \mathfrak{m}^{c} \longrightarrow \operatorname{Coker}(\alpha) \longrightarrow 0 .
$$

Obviously, there is a surjective map of the following:

$$
\operatorname{Coker}(\alpha)=Q /\left(u Q+\mathfrak{m}^{c}\right) \longrightarrow Q /\left(J+\mathfrak{q}^{[n]} Q\right)=B / \mathfrak{q}^{[n]} B .
$$

Therefore $\ell_{Q}(\operatorname{Coker}(\alpha)) \geqslant \ell_{Q}\left(B / \mathfrak{q}^{[n]} B\right)$. From (b) it follows that $B / \mathfrak{q}^{[n]} B$ has finite length over $Q$. The ideal $\mathfrak{q}$ is an $\mathfrak{n}$-primary ideal of $A$; therefore $\ell_{A}\left(B / \mathfrak{q}{ }^{[n]} B\right)$ is finite, and since $k=A / \mathfrak{n} \simeq Q / \mathfrak{m}$, it is clear that the length of $B / \mathfrak{q}^{[n]} B$ over both $Q$ and $A$ are equal. From (a) we get that $\ell_{A}\left(B / \mathfrak{q}^{[n]} B\right) \geqslant \ell_{A}\left(A / \mathfrak{q}^{[n]}\right)$. Therefore we have $\ell_{Q}(\operatorname{Coker}(\alpha)) \geqslant \ell_{A}\left(B / \mathfrak{q}^{[n]} B\right) \geqslant \ell_{A}\left(A / \mathfrak{q}^{[n]}\right)$, and from [5. (14.10)] we find that $\ell_{A}\left(A / \mathfrak{q}^{[n]}\right) \geqslant n^{t} e(A) \geqslant n^{t}$, wherein the symbol $e(A)$ is the multiplicity of $A$. Thus $\ell_{Q}(\operatorname{Coker}(\alpha)) \geqslant n^{t}$.

Let $a_{1}, a_{2}, \ldots, a_{t}$ be a minimal generator for $\mathfrak{m}$. The local ring $Q$ is regular; hence the elements $a_{j}$ for $1 \leqslant j \leqslant t$ are analytically independent in $\mathfrak{m}$. Thus we have $\nu_{Q}\left(\mathfrak{m}^{c-i}\right)=\left(\begin{array}{c}c-i+t-1 \\ t-1\end{array}\right)$ for $i \geqslant 1$. Since $u$ is not an element of $\mathfrak{m}^{d+1}$, it follows that $\operatorname{Ker}(\alpha) \subseteq \mathfrak{m}^{c-d} / \mathfrak{m}^{c}$ and hence $\ell_{Q}(\operatorname{Ker}(\alpha)) \leqslant \ell_{Q}\left(\mathfrak{m}^{c-d} / \mathfrak{m}^{c}\right)$. The following equality is clear:

$$
\ell_{Q}\left(\mathfrak{m}^{c-d} / \mathfrak{m}^{c}\right)=\sum_{i=1}^{d} \nu_{Q}\left(\mathfrak{m}^{c-i}\right) .
$$

From (2.1.1) it is clear that $\ell_{Q}(\operatorname{Ker} \alpha)=\ell_{Q}($ Coker $\alpha)$, and from (2.1.2) we find that $\ell_{Q}\left(\mathfrak{m}^{c-d} / \mathfrak{m}^{c}\right) \leqslant d\left(\begin{array}{c}c+t-2 \\ t-1\end{array}\right) \leqslant d c^{t-1}=d(t d n)^{t-1}$. Therefore $n^{t} \leqslant d^{t} t^{t-1} n^{t-1}$, and this contradicts the choice of $n$. Hence we must have $J \subseteq \mathfrak{m}^{d+1}$.

Now assume that for an ideal $I$ of $A, \ell_{Q}(Q /(J+I Q))=d$. From this we get $\mathfrak{m}^{d} \subseteq J+I Q \subseteq \mathfrak{m}^{d+1}+I Q$. Thus $\mathfrak{m}^{d}+I Q=\mathfrak{m}^{d+1}+I Q$, and by using Nakayama's Lemma, $\mathfrak{m}^{d+1} \subseteq I Q$, which yields that $J \subseteq I Q$. 
Definition 2.2. Let $(R, \mathfrak{m}, k)$ be a local ring and let $R[[X]]=R\left[\left[X_{1}, \ldots, X_{n}\right]\right]$ be the formal power series of $n$ variables over $R$. Write $A=R[[X]] / J$ for an ideal $J$ of $R[[X]]$. If the local ring $A \otimes_{R} k$ is a complete intersection, then $A$ is called a complete intersection $R$-algebra.

Let $p$ be a prime number. In the rest of this paper, we assume that all rings are of characteristic $p>0$. In the following, we recall some notions in the theory of tight closure.

Definition 2.3. Let $R$ be a ring and let $J=\left(a_{1}, \ldots, a_{t}\right)$ be an ideal of $R$. An element $x \in R$ is said to be in the tight closure of $J$, and we write $x \in J^{*}$ if there is an element $c \in R^{0}$ such that for all large $q=p^{n}$ we have $c x^{q} \in J^{[q]}$, wherein $R^{0}$ is the complement of the union of all minimal primes of $R$ and $J^{[q]}=\left(a_{1}^{q}, \ldots, a_{t}^{q}\right)$. An ideal $J$ is called tightly closed if $J=J^{*}$. The ring $R$ is called $F$-rational if every parameter ideal of $R$ is tightly closed.

To state the main theorem of this paper, we will make use of the following simple lemma, the proof of which is omitted.

Lemma 2.4. Let $I$ be an ideal of $R$; then $\left(I^{*}\right)^{n} \subseteq\left(I^{n}\right)^{*}$ for $n \geqslant 1$.

Theorem 2.5. Let $\eta:(A, \mathfrak{n}) \rightarrow(Q, \mathfrak{m})$ be a local ring homomorphism such that $\operatorname{dim} A=\operatorname{dim} Q=t, Q$ is regular, and the induced homomorphism $\bar{\eta}: A / \mathfrak{n} \rightarrow Q / \mathfrak{m}$ is an isomorphism. Let $\mathfrak{q}$ be an ideal of $A$ generated by a system of parameters and let $\theta: Q \rightarrow B$ be a surjective ring homomorphism such that $\ell_{Q}\left(B / \mathfrak{q}^{*} B\right)=d$. Assume that for an integer $q=p^{n}>d^{t} t^{t-1}$ the ideal $\mathfrak{q}^{[q]}$ is tightly closed; then

(a) $\ell_{A}\left(B / \mathfrak{q}^{[q]} B\right)=\ell_{Q}\left(B / \mathfrak{q}^{[q]} B\right)<\infty$.

(b) Let $A$ be an equidimensional homomorphic image of a Cohen-Macaulay ring. If $\ell_{Q}\left(B / \mathfrak{q}^{[q]} B\right) \geqslant \ell_{A}\left(A / \mathfrak{q}^{[q]}\right)$, then the canonical homomorphism $\pi: Q / \mathfrak{q} Q \rightarrow B / \mathfrak{q} B$ is an isomorphism between complete intersection rings.

Proof. (a) We only have to prove that $\ell_{Q}\left(B / \mathfrak{q}^{[q]} B\right)<\infty$. The equality was shown in the proof of Lemma 2.1. Assume that $B=Q / J$, in which $J=\operatorname{Ker} \theta$. From the assumptions it follows that $\mathfrak{m}^{d} \subseteq J+\mathfrak{q}^{*} Q$. Therefore we have the next inclusions $\mathfrak{m}^{t q d} \subseteq\left(J+\mathfrak{q}^{*} Q\right)^{t q} \subseteq\left(J+\left(\mathfrak{q}^{*}\right)^{t q} Q\right)$. Lemma 2.4 implies that $\mathfrak{m}^{t q d} \subseteq J+\left(\mathfrak{q}^{t q}\right)^{*} Q$. Since $\mathfrak{q}$ is generated by $t$ elements, it is easy to see that $\mathfrak{q}^{t q} \subseteq \mathfrak{q}^{[q]}$. This yields that $\mathfrak{m}^{t q d} \subseteq J+\left(\mathfrak{q}^{[q]}\right)^{*} Q$, and from our assumptions we have $\mathfrak{m}^{t q d} \subseteq J+\mathfrak{q}^{[q]} Q$ and this proves that $\ell_{Q}\left(B / \mathfrak{q}^{[q]} B\right)<\infty$.

(b) In part (a) it was shown that $\mathfrak{m}^{t q d} \subseteq J+\mathfrak{q}^{[q]} Q$. From our assumptions, we have $\ell_{Q}\left(B / \mathfrak{q}^{[q]} B\right) \geqslant \ell_{A}\left(A / \mathfrak{q}^{[q]}\right)$ and $\ell_{Q}\left(B / \mathfrak{q}^{*} B\right)=d$. Thus from Lemma 2.1, it follows that $J \subseteq \mathfrak{q}^{*} Q$. This shows that the canonical homomorphism $\pi^{*}: Q / \mathfrak{q}^{*} Q \rightarrow B / \mathfrak{q}^{*} B$ is an isomorphism. Now let $A$ be an equidimensional ring which is a homomorphic image of a Cohen-Macaulay ring. In this case, since $\mathfrak{q}^{[q]}$ is generated by a system of parameters and it is tightly closed, it follows from [4, (4.2.e)] that $A$ is $F$-rational and hence $\mathfrak{q}=\mathfrak{q}^{*}$. Now it is easy to see that $\pi$ is an isomorphism between complete intersection rings.

Remark 2.6. Let $(\mathcal{O}, \mathfrak{m}, k)$ be a complete local ring with finite residue field $k$ and let $\mathcal{D}$ be a minimal deformation theory. Let $R$ and $T$ be the universal deformation and Hecke $\mathcal{O}$-algebras associated to $\mathcal{D}$, respectively, and let $\varphi=\varphi_{\mathcal{D}}$ be the homomorphism described in Section 1. In the appendix of [7] for a simplification of some arguments in Section 3 of [7] and Chapter 3 of [8], Taylor and Wiles use an 
algebraic result of Faltings to re-prove that $\varphi$ is an isomorphism between complete intersection algebras. We prove Proposition 2.8, which contains corresponding results due to Taylor and Wiles, and Faltings without the assumption of finiteness of $k$. Our method of proving the above result is totally different from their methods. In the following we refine the definition of a (level) $n$-structure due to Wiles and Faltings in [7].

Definition 2.7. Let $(\mathcal{O}, \mathfrak{m}, k)$ be a local $\operatorname{ring}$ and let $\mathcal{O}[[Y]]=\mathcal{O}\left[\left[Y_{1}, \ldots, Y_{t}\right]\right]$. Set $\mathfrak{q}=\left(Y_{1}, \ldots, Y_{t}\right)$ and assume that for an integer $n \geqslant 1$ we have a commutative diagram of local $\mathcal{O}$-algebras as the following in which $\varphi$ is surjective and $T$ is a finite free $\mathcal{O}$-module:

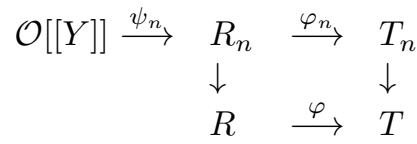

By a level $n$-structure we mean the above diagram with the following properties:

(1) There is a surjective homomorphism $\lambda_{n}: \mathcal{O}\left[\left[X_{1}, X_{2}, \ldots, X_{t}\right]\right] \longrightarrow R_{n}$.

(2) The ring homomorphism $\varphi_{n}$ is surjective.

(3) From the vertical homomorphisms we get the isomorphisms $R_{n} / \mathfrak{q} R_{n} \longrightarrow R$ and $T_{n} / \mathfrak{q} T_{n} \longrightarrow T$.

(4) The ring $T_{n} / \mathfrak{q}^{\left[p^{n}\right]} T_{n}$ is finite and free as a module over $\mathcal{O}[[Y]] / \mathfrak{q}^{\left[p^{n}\right]}$.

Proposition 2.8. Let $(\mathcal{O}, \mathfrak{m}, k)$ be an $\mathfrak{m}$-adic complete local ring and let $k \geqslant 1$ be an integer such that for each integer $n \geqslant k$ there is a level $n$-structure. Then $\varphi$ is an isomorphism between complete intersection $\mathcal{O}$-algebras.

Proof. To simplify we write $\widehat{\square}=\square \otimes_{\mathcal{O}} k, \mathfrak{q}_{n}=\mathfrak{q}^{\left[p^{n}\right]}$ and $\mathcal{O}[[Z]]=\mathcal{O}\left[\left[Z_{1}, Z_{2}, \ldots, Z_{t}\right]\right]$ for a ring of formal power series with $t$ variables. Now choose $n \geqslant k$ such that $p^{n}>t^{t-1} d^{t}$. From (1) there is a surjective homomorphism $\mathcal{O}[[X]] \rightarrow R_{n}$. Since $\mathcal{O}$ is $\mathfrak{m}$-adic complete, it follows that $\mathcal{O}[[X]]$ is $(\mathfrak{m}[[X]]+(X))$-adic complete. Thus from [1, (7.16)(a)] we can lift $\psi_{n}$ to a ring homomorphism $\eta: \mathcal{O}[[Y]] \longrightarrow \mathcal{O}[[X]]$ such that $\eta(\mathfrak{m}[[Y]]) \subseteq \mathfrak{m}[[X]]$. Therefore $\eta$ induces a local ring homomorphism $\widehat{\eta}: k[[Y]] \longrightarrow k[[X]]$. From (1), (2) and (3) we find that there is a surjective ring homomorphism such as the following:

$$
\alpha: k[[X]] / \mathfrak{q} k[[X]] \longrightarrow \widehat{T_{n}} / \mathfrak{q} \widehat{T_{n}} \simeq \widehat{T} .
$$

Since $T$ is a finite free $\mathcal{O}$-module, from (3) it follows that $\widehat{T_{n}} / \mathfrak{q} \widehat{T_{n}}$ is a finite vector space over $k$. We may assume that the length of $\widehat{T_{n}} / \mathfrak{q} \widehat{T_{n}}$ over $k[[X]]$ is equal to $d$. From the fourth property it is clear that the length of $\widehat{T_{n}} / \mathfrak{q}_{n} \widehat{T_{n}}$ over $k[[Y]]$ is equal to or greater than the length of $k[[Y]] / \mathfrak{q}_{n} k[[Y]]$.

By writing $A=k[[Y]], Q=k[[X]]$ and $B=\widehat{T_{n}}$, from Theorem 2.5] it follows that $\alpha$ is an isomorphism between complete intersection rings.

On the other hand, $\varphi_{n}$ is surjective. From this and the third property we have the following surjective homomorphisms:

$$
\begin{gathered}
\beta: k[[X]] / \mathfrak{q} k[[X]] \longrightarrow \widehat{R_{n}} / \mathfrak{q} \widehat{R_{n}} \\
\widehat{\varphi}: \widehat{R_{n}} / \mathfrak{q} \widehat{R_{n}} \longrightarrow \widehat{T_{n}} / \mathfrak{q} \widehat{T_{n}}
\end{gathered}
$$

such that $\alpha=\widehat{\varphi} \beta$. It was shown that $\alpha$ is an isomorphism. Thus from (3) it is clear that $\widehat{\varphi}$ is an isomorphism between $\widehat{R}$ and $\widehat{T}$ of complete intersection rings. Since $T$ is a finite free $\mathcal{O}$-module and $\varphi$ is surjective, Nakayama's Lemma implies 
that $\varphi$ is an isomorphism. It is obvious that the local $\operatorname{ring} T$ is a homomorphic image of $\mathcal{O}[[X]]$. Since $\widehat{T}$ is a complete intersection ring, it follows that $T$ and $R$ are complete intersection $\mathcal{O}$-algebras.

\section{ACKNOWLEDGMENTS}

The author is grateful to Sean Sather-Wagstaff, Irena Swanson and Siamak Yassemi for their useful comments on this paper. He is also deeply grateful to the referee for the careful reading of this work and useful suggestions.

\section{REFERENCES}

1. D. Eisenbud, Commutative algebra with a view toward algebraic geometry, Springer, 1995. MR 1322960 (97a:13001)

2. M. Hochster, C. Huneke, Tight closure, invariant theory, and the Briançon-Skoda theorem, J. Amer. Math. Soc. 3 (1990), 31-116. MR1017784 (91g:13010)

3. M. Hochster, C. Huneke, Tight closure of parameter ideals and splitting in module-finite extensions, J. Algebraic Geom. 3 (1994), no. 4, 599-670. MR.1297848 (95k:13002)

4. C. Huneke, Tight closure and its applications, with an appendix by Melvin Hochster. CBMS Regional Conference Series in Mathematics, 88, Amer. Math. Soc., Providence, RI, 1996. MR 1377268 (96m:13001)

5. H. Matsumura, Commutative ring theory, Cambridge Studies in Advanced Mathematics, 8, Cambridge University Press, Cambridge, 1989. MR.1011461 (90i:13001)

6. K. A. Ribet, Galois representations and modular forms, Bull. Amer. Math. Soc. (N.S.) 32 (1995), no. 4, 375-402. MR1322785 (96b:11073)

7. R. Taylor, A. Wiles, Ring-theoretic properties of certain Hecke algebras, Ann. of Math. (2) 141 (1995), 553-572. MR1333036 (96d:11072)

8. A. Wiles, Modular elliptic curves and Fermat's last theorem. Ann. of Math. (2) 141 (1995), no. 3, 443-551. MR.1333035 (96d:11071)

School of Mathematics, Institute for Research in Fundamental Sciences (IPM), P.O. Box 19395-5746, TeHran, Iran

E-mail address: sharif@ipm.ir

URL: http://www.ipm.ac.ir/IPM/people/personalinfo.jsp?PeopleCode=IP0400060 